\title{
An optimal algorithm based on the solution to the coarse-grained arc consistency algorithms of the Constraint satisfaction Problems
}

\author{
Gang Yang, Huifeng Li, Can Cao, Siyuan Chen, and Yubo Zhao \\ College of Computer Science and Technology \\ Jilin University \\ Changchun, China, 130012
}

\begin{abstract}
Constraint satisfaction problem are widely used in the artificial intelligence field. We study the coarse-grained arc consistency algorithms of the Constraint satisfaction Problems. We find that variables whose degrees or domains are 1 have invalid revises which can be removed in the process. We demonstrate that these revises are redundant. We also provide an improved method to avoid the redundancy. The improved framework of the constraint problemsAC3 algorithms contain the ignorance of degree is 1, and do main is 1.The improved framework can be used on all of the coarse-grained arc consistency algorithms. The result of the test shows that we can save up to $65 \%$ revise times and $10 \%$ execution time after using the improved algorithms AC3 algorithms containing the ignorance of degree is 1 , and domain is 1.
\end{abstract}

Keywords: Constraint satisfaction problem; Maintaining arc consistency; the Coarse-grained algorithms; Revise; degree is 1; domain is 1

\section{INTRODUCTION}

Many problems in our daily life can be solved by establishing a constraint satisfaction problem (CSP) ${ }^{[1]}$ model, for instance, the resources allocation and the scheduling problem. Also, applications like the spatial graphic representation, the design constraint of hardware and the check plot, are all simulations of the CSP. This characteristic has made CSP widely use in the artificial intelligence field. A solution to CSP problem is to assign values to all the variables in their domains, making the instantiated variables satisfy the given constraints. While it was recognized that solving CSPs was in general NP-hard, a variety of analytical techniques were brought to bear to evaluate, predict or compare algorithm performance and relate problem complexity to problem structure. ${ }^{[1]}$ Among those techniques, backtracking algorithm (BT) is a complete algorithm. ${ }^{[2]}$ While because the basic BT is not very efficient, usually, people use constraint propagation before and during search to reduce the scale of problems, eliminating some of the inexistent variables to narrow the search space and cut down search times to improve the efficiency. Arc Consistency (AC) is the earliest and the most widely used technique among all the existed constraint propagation. Maintaining Arc Consistency (MAC) is regarded as the most sufficient method to solve large-scale of hard problems. ${ }^{[4]}$ There are two types of AC classified by different types of constraint propagation, one is the coarse-grained arc consistency algorithms, which propagate on the arc, and the other one is fine-grained arc consistency algorithms, which propagate among the variables. ${ }^{[5]}$ Using less cost of space, the coarse-grained algorithms are more widely used in the practice. Mackworth (1977) raised the AC3, a classical arc consistency coarse-grained algorithm, which points out that the coarse-grained arc consistency algorithm is combined of two parts: basic framework and revise.

This paper analyses the process of the coarse-grained arc consistency algorithms, and finds that there are insufficient correction check in the arc when the degree or value equals 1 . We testify that this kind of revise which appears rarely in the preprocessing of the arc consistency and mostly in the processing is redundant. We provide a method called Ignore for a degree and for a domain (IDD), avoiding the redundant revise and improving the basic framework of the coarse-grained arc consistency algorithms. The improved framework AC3 IDD can be used to better all the coarse-grained MAC algorithms presented. Finally, we apply our method into the recently popular coarse-grained arc consistency algorithms, MAC3rm. The result of the experiment shows that the improved algorithms can save up to $65 \%$ of the revise times, and raise the efficiency of solving to $10 \%$.

\section{PRELIMINARIES}

Definition 1(Constraint Satisfaction Problem, CSP) ${ }^{[1]} \mathrm{A}$ CSP $\mathrm{P}$ is a triple $\mathrm{P}=\langle\mathrm{X}, \mathrm{D}, \mathrm{C}>$ where $\mathrm{X}$ is an n-tuple of variables $X=\{x 1, x 2, \ldots, x n\}, D$ is a corresponding n-tuple of domains $\mathrm{D}=\{\mathrm{D} 1, \mathrm{D} 2, \ldots, \mathrm{Dn}\}, \mathrm{Di} \in \mathrm{D}$ is the finite domain of $\mathrm{xi}$, when $\mathrm{xi} \in \mathrm{X}, \mathrm{C}$ is a t-tuple of constraints $\mathrm{C}=\{\mathrm{C} 1, \mathrm{C} 2, \ldots, \mathrm{Ck}\}$. Any $\mathrm{C} j \in \mathrm{C}$ represents the constraint relation of the variables.

Definition 2 (arc consistency, AC) ${ }^{[6]}$ For a given binary CSP P, $(\mathrm{x}, \mathrm{Cxy})$ is an arc in $\mathrm{P}$, if any a, of which domain is $\mathrm{x}$, can find $a \mathrm{~b}$, of which domain is $y$, to make $(a, b)$ satisfy Cxy, then $\operatorname{arc}(\mathrm{x}, \mathrm{Cxy})$ is arc consistency. Only when each arc of the CSP is $\mathrm{AC}$, can the CSP be AC.

Values which cannot find supporting from their adjacent arcs have to be removed, when processing the arc constraint check. If all the values in the domain of a variable are removed, then the problem $\mathrm{P}$ has no solution, return arc consistency check 
fail.

Maintaining Arc Consistency(MAC) is a highly efficient basktrack search algorithms for solving CSP problems. MAC is to embed AC algorithms under the framework of BT algorithms. In the search process maintaining arc consistency situation, using $\mathrm{AC}$ algorithms to constraint propagation each time after assigning a value to a variable, if we get an arc consistency situation, then the assignment is successful; otherwise, the assignment is failed and either select the next assignment or backtrack. Currently used MAC is the MAC3rm algorithms which are coarse-grained algorithms rose by Lecoutre in 2007.

Definition 3 Degree TheDegree of a variable is the number of other variables when there are constraints between the variable and the others.

Definition 4 Domain Eachdomain has its own domain. We concern not only the value of the domain, but also whether the domain is 1 .

Algorithm 1 is the basic framework of the coarse-grained AC algorithms. $Q$ is the collection need to be propagated, keeping all the arcs that are yet to be checked; initialize $Q$ is to initialize the collection to be propagated, if AC algorithms are used in the preprocessing, then we need to add all the arcs in the problem into $Q$ to initialize $Q$. In the process of arc consistency check, if the domain of any variables changed, then we have to add all the arcs pointing to that variable to $Q$ and check the arcs again. If the domain of any variable is empty, then return check fail; otherwise when $\mathrm{Q}$ is empty, return arc consistency check successful. In algorithm 2, function revise is the check and revise process of the coarse-grained algorithm. $D x$ is the finite domain of $x$, Revise $(x, c)$ is to find supports for all the unremoved value in the domain of $x$ on the arc. All the AC algorithms mentioned below are coarse-grained arc consistency algorithms.

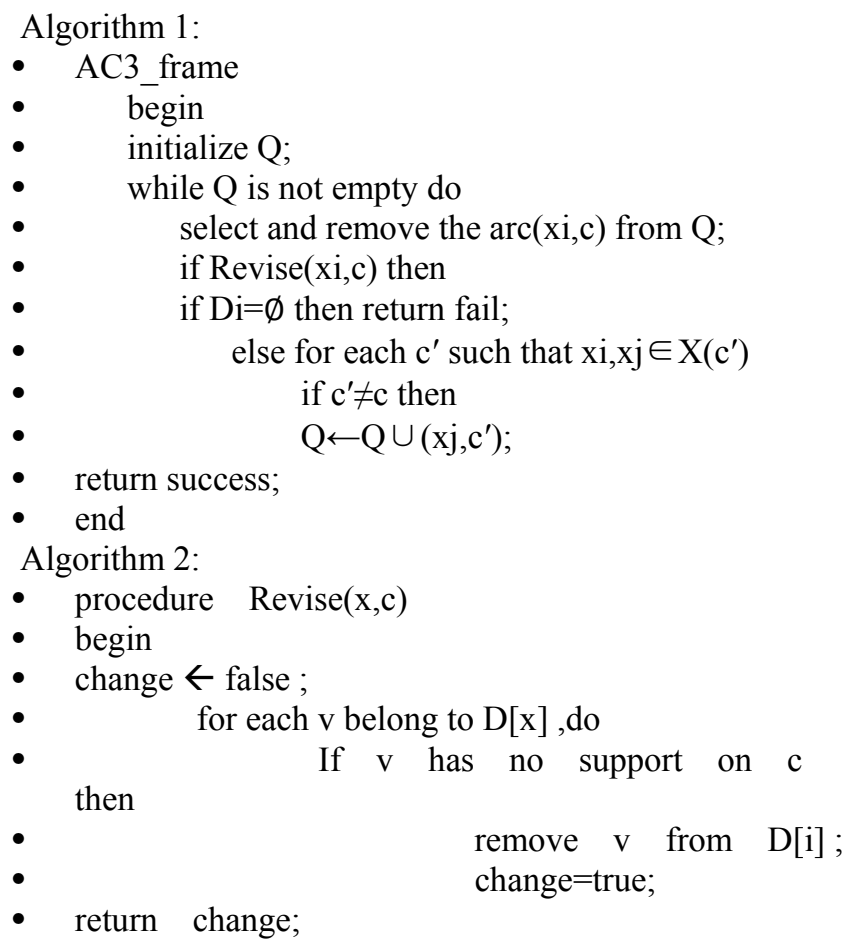

- $\quad$ End

\section{REDUNDANCY CHECK}

Before starting the MAC algorithms, we need to process the arc consistency preprocessing through $\mathrm{AC}$ algorithms. If the preprocessing failed, the problem will have no solution; otherwise, we can begin to backtrack search. Therefore, the problem is in an AC situation before the backtrack search. Each time we assign a value, we need to process the constraint propagation through $\mathrm{AC}$ algorithms. If the $\mathrm{AC}$ algorithms are successful, then we can do the next assignment; otherwise, the backtrack happens. Thus, in the process of MAC algorithms search, the problem must be in an AC situation before assignment. To assign value $a$ to variable $x$ is to remove all the values in the domain of $x$ except $a$. Therefore, each time after assignment, only the domain of the current variable will change. We only need to use all the arcs pointing to $x$ to initiate the collection $Q$ to be propagated, and then execute AC algorithms to process the constraint propagation

Further, when the degree of a variable is 1 , we can make sure whether the variable is arc consistency by checking the related variables in the collection because of the bidirectional support of the constraints. Therefore, the check of this variable is redundant, and ignorance of the variable will not affect the solver. We can then ignore the variables whose degrees are 1. All the related variables need to be compared with a certain value of the variable, when the domain of the variable is 1 . The problem of comparing different values does not exist. Thus, ignorance of the variables whose domains are 1 will not affect the solver, when the preprocessing has passed.

As a result, the idea that a new variable whose degree is 1 can be generated when certain variable whose domain is 1 is ignored and its related variables are also ignored comes to our mind. Because of the constraint propagation, the solution of the problem can be greatly simplified. We then design a new algorithm AC3 rm IDD:

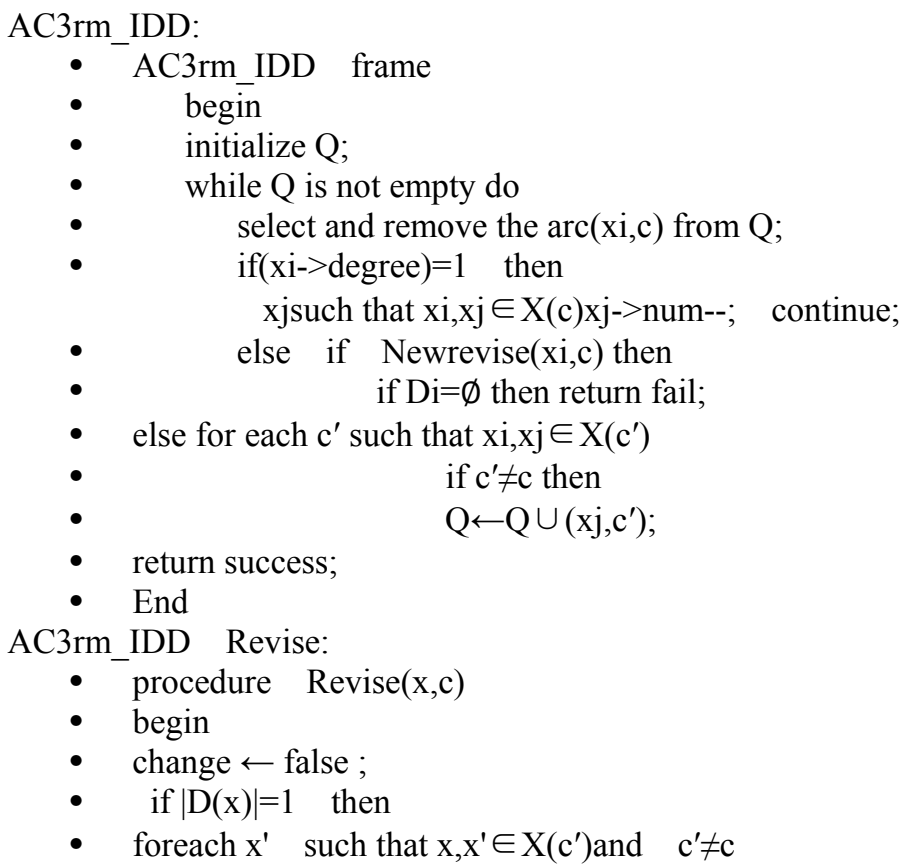


$\mathrm{X}^{\prime}->$ num--; $\quad$ return false

- else for each v belong to $\mathrm{D}[\mathrm{x}]$,do

- If $\mathrm{V}$ has no support on $\mathrm{c}$ then

- change $\leftarrow$ true;

- return change;

- End

In Figure 1, the ovals represent the initial domains of certain variables, the lines between values show that the two values satisfy the constraint. Before solving the problem, the result of the arc consistency preprocessing is showed in Figure 2. During the process, we first assign 0 to $x 0$, add it into $Q$, andthen execute $\mathrm{AC}$ algorithms revise $(x 0, c 0)$, after that, 0 is removed from the domain of $x 2$, and the assignment of $(x 0,0)$ is successful. Next step, $x 0$ can be ignored because its domain has changed to 1 and there is no need to put it into the collection. Meanwhile, $x 3$ can also be ignored because its degree becomes 1 after minus by 1 . Therefore, we can avoid the repetition of algorithm revise andsave the processing time. Figure 3 shows the process of solving the constraint problems.

Fig. 1 Previous constraint problem

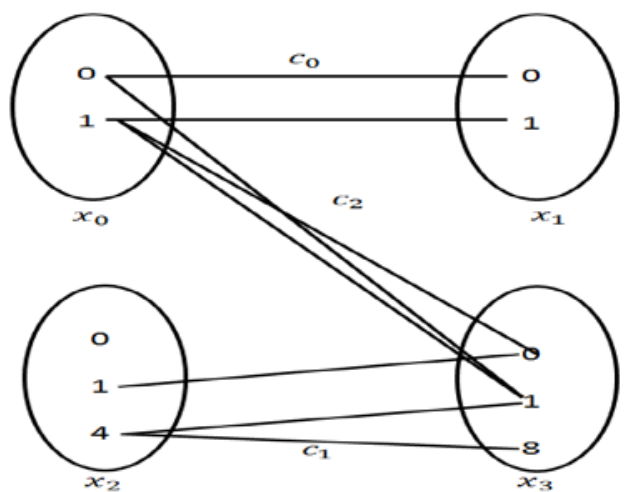

Fig. 2 Arc consistency preprocessed

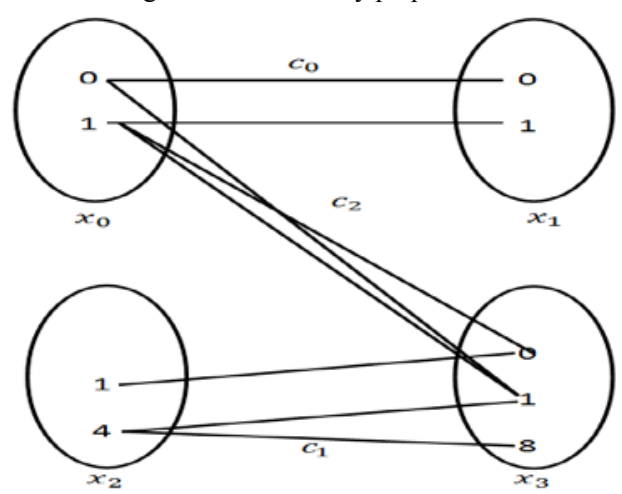

Fig. 3 Solution of constraint problem

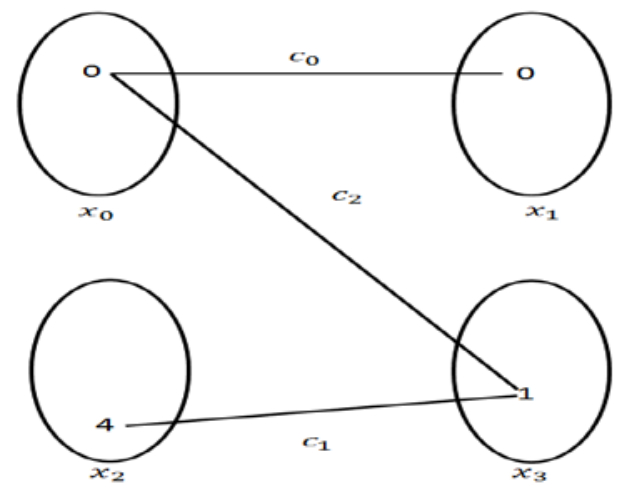

IV. RELATED WORK AND DISCUSSION

We find that we can determine the variables in the arc before we get the arc in the collection by AC3rm algorithms and analyse. If the degree of the variable is 1 , then only one relation is between the variable and other variables (the number of the constraint related to the variable is only one). According to the bidirectional support of the constraints, we can determine whether the arc is consistent when judging the variable on the other side of the constraint of the arc. So the removal of the revises can be remained, but putting back all the arcs which are related to the variable on the other side of the constraint can be deleted. That is the ignorance of the degree of 1 . This method reduces the additions to the arcs in the collection, and simplifies the solution. It is the same with the checking function in AC3rm algorithms. According to the bidirectional support of the constraints, we can determine whether the arc is consistent when checking the variable on the other side of the constraint of the arc. So the check can be ignored and the efficiency of solution is improved. Also, all the variables related to the variable need to minus 1 after the ignorance of the variable whose degree is 1 . The ignorance of 1 degree variables can reduce the process of supporting check, save the time of solution, and optimize the whole process. In summary, we combine the theories of degree is 1 and domain is 1 . Degree is 1 can improve domain is 1 . Therefore, the theory can increase the solving efficiency of the problems and reach the optimization effect. However, if the concepts of degree is 1 and domain is 1 are added, we will inevitably add related data structures and functions to maintain the data structure. This will cost more spending which can impede the improvement of solving time. The reduction of the times of constraint check illustrates that it is correct to add the theory of degree is 1 and domain is 1 into the basic framework of the coarse-grained algorithm. We believe that as long as the maintenance of the data structures is briefly enough, the skill of process is of high quality, the time of the CPU execution will be shortened largely, and the save of time is very obvious.

\section{TEST RESULT}

We use two different algorithms to test the improvement of efficiency of the coarse-grained algorithms by IDD method. The basic algorithm is the currently international prevalent coarse-grained algorithms Mac3rm; Mac3rm_IDD is the improved algorithm which adds degree is 1 and domain is 1 skill to the basic of Mac3rm. Using IDD to improve Mac3rm, Mac3rm IDD is a new method that used the framework of AC3 frame_IDD to improve the framework of Mac3rm. The use 
case of test is the benchmark in the standard use case. Figure 4 is the line chart of the contrast of CPU execution time between MAC3rm and Mac3rm IDD. Figure 5 is the line chart of the times of revises between MAC3rm and Mac3rm_IDD. The result of the test shows that comparing those with the coarse-grained arc consistency algorithm MAC3rm, the efficiency of CPU improved by $10 \%$ and the times of revises reduced by $65 \%$ after adding the IDD method Mac3rm_IDD.

Table 1

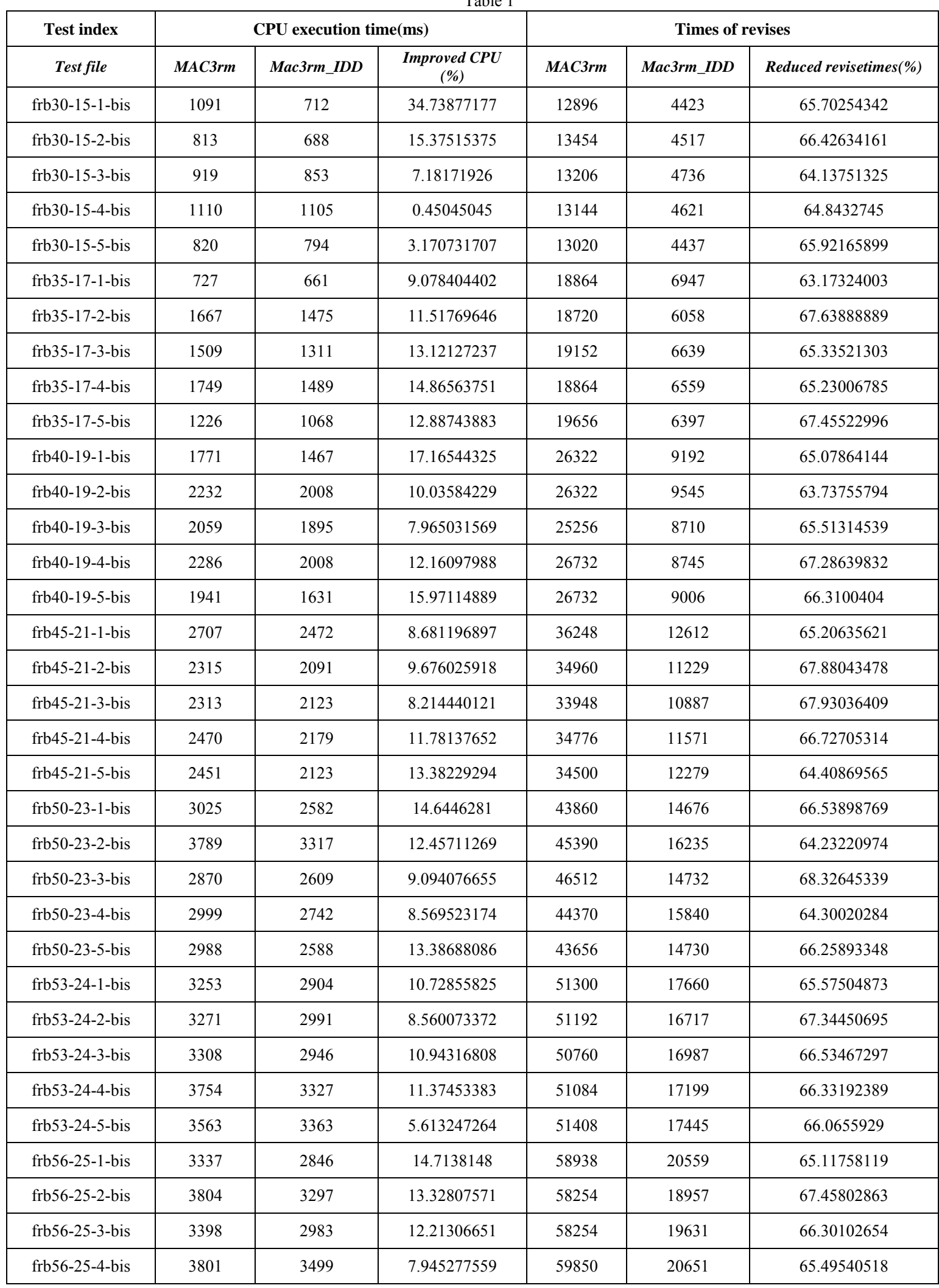




\begin{tabular}{|l|l|l|l|l|l|l|}
\hline frb56-25-5-bis & 2718 & 2522 & 7.211184695 & 58368 & 20524 & 64.83689693 \\
\hline frb59-26-1-bis & 4415 & 3979 & 9.875424689 & 66720 & 22151 & 66.80005995 \\
\hline frb59-26-2-bis & 4224 & 3551 & 15.93276515 & 65880 & 22341 & 66.08834244 \\
\hline frb59-26-3-bis & 4049 & 3543 & 12.49691282 & 65640 & 21899 & 66.6377209 \\
\hline frb59-26-4-bis & 4010 & 3702 & 7.680798005 & 67080 & 20985 & 68.71645796 \\
\hline frb59-26-5-bis & 4224 & 3849 & 8.877840909 & 64680 & 21985 & 66.00958565 \\
\hline
\end{tabular}

Fig. 4 Comparison of the CPU execution time between MAC3rm and Mac3rm IDD

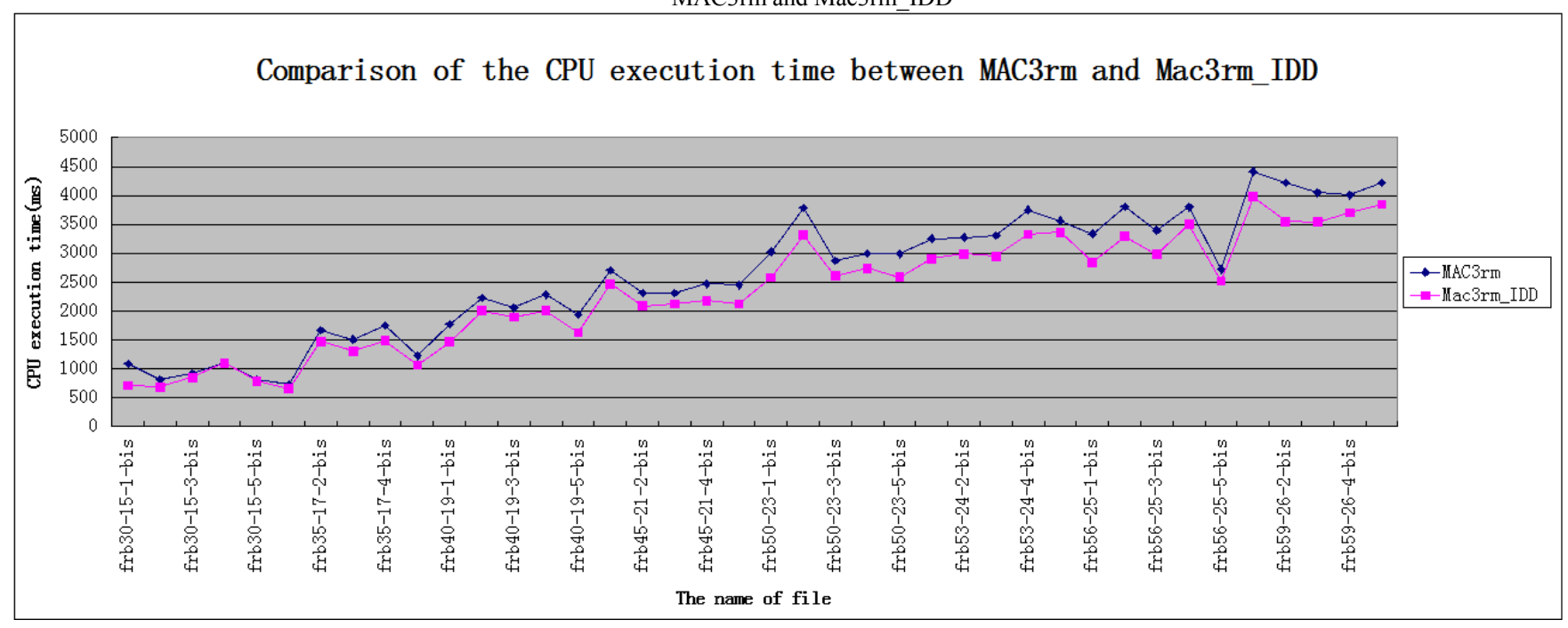

Fig. 5 Comparison of the revise times between MAC3rm and Mac3rm_IDD

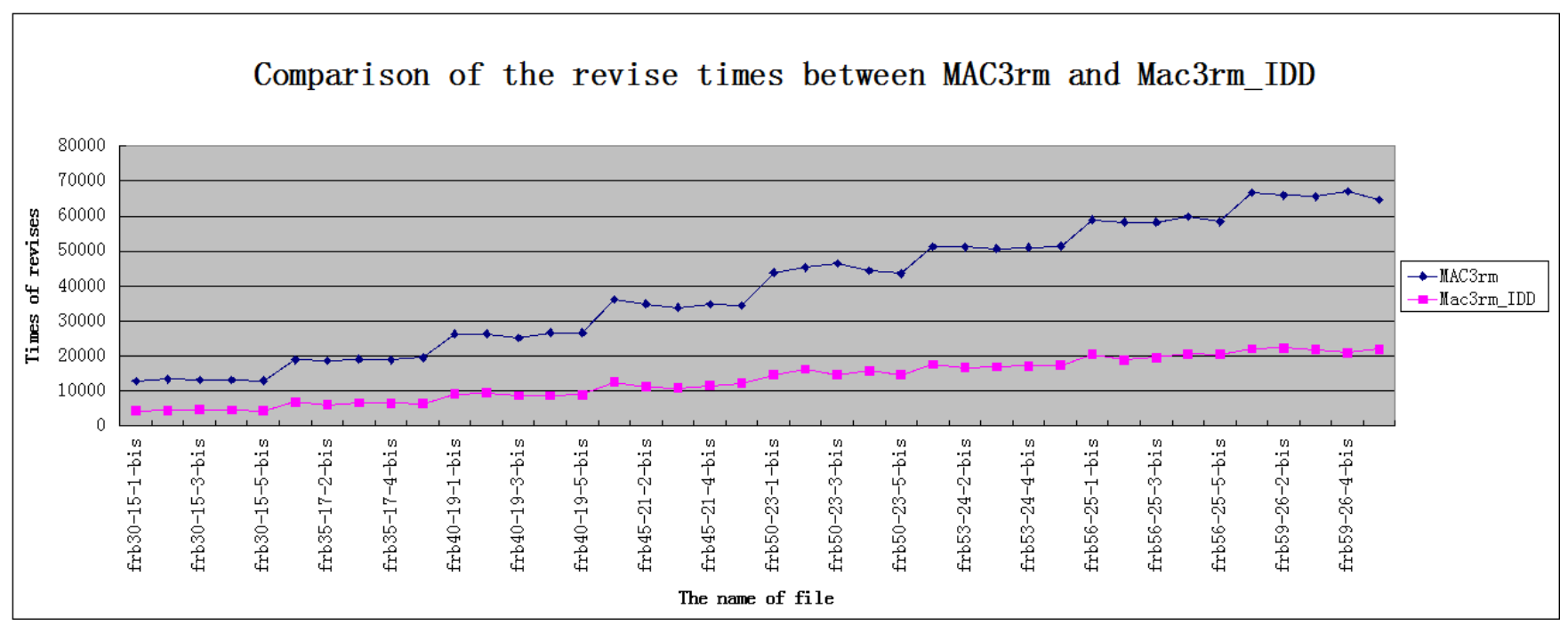




\section{CONCLUSION}

Revise is the essential core part of the coarse-grained arc consistency algorithms. The worst time complexity of the execution of revise is $O\left(d^{2}\right)$. Therefore, avoiding the useless revise can increase the efficiency of algorithms. Most of the studies of the coarse-grained algorithms are concentrated on the improvement of the revise, and few of them have improved the framework of the algorithms. We study the execution of the coarse-grained maintaining arc consistency algorithms, finding that there are some invalid revises in them. These revises are mainly focused on the variables whose degree is 1 or domain is 1 . By using the bidirectional support of the constraints, we can ignore those variables whose degree or domain is 1 , reducing the added arcs in the collection and the times of revise. Therefore, the workload can be reduced, and the previous algorithms are optimized.

\section{REFERENCES}

[1]. Freuder EC, Mackworth AK. Constraint satisfaction: An emerging paradigm. Rossi F, van Beek P, Walsh $T$, eds. Handbook ofConstraint Programming.Amsterdam:Elservier,2006.13-27.[doi: 10.1016/S1574-6526(06)80006-4]

[2]. vanBeek P. Backtracking search algorithms. In: Rossi F, van Beek P, Walsh T, eds. Handbook of Constraint Programming.Amsterdam: Elservier, 2006. 85-134. [doi: 10.1016/S1574-6526(06)80008-8]

[3]. Bessière C. Constraint propagation. Rossi $\mathrm{F}$, van Beek $\mathrm{P}$, Walsh T, eds. Handbook of Constraint Programming. Amsterdam:Elservier, 2006. 29-84.

[4]. Sabin D, Freuder EC. Contradicting conventional wisdom in constraint satisfaction. In: Cohn AG, ed. Proc. of the 11th EuropeanConf. on Artificial Intelligence. Amsterdam: John Wiley \& Sons, 1994. 125-129.

[5]. Bessière C, Regin JC, Yap RHC, Zhang YL. An optimal coarse-grained arc consistency algorithm. Artificial Intelligence, 2005,

165(2):165-185. [doi: 10.1016/j.artint.2005.02.004]

[6]. Mackworth AK. Consistency in networks of relations. Artificial Intelligence, 1977,8(1):99-118. [doi: 10.1016/0004-3702(77)90007-8] 